\title{
Effects of sleep deprivation on central auditory processing
}

\author{
Paulo Breno Noronha Liberalesso ${ }^{1,2}$, Karlin Fabianne Klagenberg D'Andrea', Mara L Cordeiro ${ }^{3,4,5^{*}}$, \\ Bianca Simone Zeigelboim¹, Jair Mendes Marques ${ }^{1}$ and Ari Leon Jurkiewicz ${ }^{1}$
}

\begin{abstract}
Background: Sleep deprivation is extremely common in contemporary society, and is considered to be a frequent cause of behavioral disorders, mood, alertness, and cognitive performance. Although the impacts of sleep deprivation have been studied extensively in various experimental paradigms, very few studies have addressed the impact of sleep deprivation on central auditory processing (CAP). Therefore, we examined the impact of sleep deprivation on CAP, for which there is sparse information. In the present study, thirty healthy adult volunteers (17 females and 13 males, aged $30.75 \pm 7.14$ years) were subjected to a pure tone audiometry test, a speech recognition threshold test, a speech recognition task, the Staggered Spondaic Word Test (SSWT), and the Random Gap Detection Test (RGDT). Baseline (BSL) performance was compared to performance after 24 hours of being sleep deprived (24hSD) using the Student's t test.

Results: Mean RGDT score was elevated in the $24 \mathrm{hSD}$ condition $(8.0 \pm 2.9 \mathrm{~ms}$ ) relative to the BSL condition for the whole cohort $(6.4 \pm 2.8 \mathrm{~ms} ; \mathrm{p}=0.0005)$, for males $(p=0.0066)$, and for females $(p=0.0208)$. Sleep deprivation reduced SSWT scores for the whole cohort in both ears [(right: BSL, $98.4 \% \pm 1.8 \%$ vs. SD, $94.2 \% \pm 6.3 \%$. $p=0.0005$ ) (left: BSL, $96.7 \% \pm 3.1 \%$ vs. SD, $92.1 \% \pm 6.1 \%, p<0.0001$ )]. These effects were evident within both gender subgroups [(right: males, $p=0.0080$; females, $p=0.0143$ )(left: males, $p=0.0076$; females: $p=0.0010$ ).
\end{abstract}

Conclusion: Sleep deprivation impairs RGDT and SSWT performance. These findings confirm that sleep deprivation has central effects that may impair performance in other areas of life.

Keywords: Central auditory processing, Sleep, RGDT, SSWT

\section{Background}

Sleep is a reversible state of consciousness characterized by the temporary suspension of perceptual/sensory phenomena and voluntary motor activity that naturally occurs at regular intervals, alternating with wakefulness. Although the precise biological functions of sleep have yet to be resolved, it is clear that sleep is vital to mammalian survival [1]. Furthermore, sleep plays an important role in learning and memory consolidation [2,3]. Inadequate sleep is a growing problem worldwide that affects healthy individuals as well as people with various disorders $[4,5]$. The lifestyles of modern societies are increasingly associated with stress and, consequently, poor

\footnotetext{
* Correspondence: mcordeiro@mednet.ucla.edu

${ }^{3}$ Department of Neuropsychopharmacology, Pelé Little Prince Research Institute, Curitiba, Brazil

${ }^{4}$ Faculdades Little Prince, Curitiba, Brazil

Full list of author information is available at the end of the article
}

sleep quality. Furthermore, reductions in the total hours of sleep obtained have tremendous impacts on various bodily systems. Reduction in hours slept has been shown to have negative effects on the immune, metabolic, and endocrine systems [6]. For example, 24.5 hours of sleep deprivation was recently shown to increase blood pressure in healthy normotensive adults [7]. And, although the etiology of mood and behavioral disorders is complex and not yet fully understood, a clear link between sleep deprivation and psychiatric conditions has been established [8,9].

Although the impact of sleep deprivation has been studied extensively in various experimental paradigms from basic animal research, such as the effects of SD on hippocampal neurogenesis [10], to sophisticated human imaging studies [11], there have been very few studies that have addressed the impact of sleep deprivation on central auditory processing (CAP). CAP can be

\section{Biomed Central}


understood as a set of neurophysiological and neurochemical mechanisms that occur in the auditory system in response to acoustic stimuli. Cognitively, CAP is critical for language comprehension and is responsible for sound localization and lateralization, sound discrimination, auditory pattern recognition, and the temporal aspects of hearing (including auditory masking, resolution, integration, and ordering), as well as for the ability to negotiate competing or degraded acoustic signals [12].

Central auditory processing disorder (CAPD) is characterized by listening difficulties despite a normal audiogram. Sleep deprivation impacts several functions associated with prefrontal cortex (PFC) activity [13,14] and auditory temporal resolution is a perceptual function associated with PFC activity [15]. Thus, it is reasonable to posit that sleep deprivation may impair CAP through circuits involving the PFC.

The aim of the present study was to evaluate the impact of sleep deprivation for a period of 24 hours on CAP in neurologically and psychologically healthy adults. CAP in a baseline control state and a 24-hour sleep-deprived state (24hSD) was assessed using the Random Gap Detection Test (RGDT) and the Staggered Spondaic Word Test (SSWT). The RGDT measures temporal resolution processing (auditory timing) abilities in the auditory domain. The SSWT measures recognition of overlapping sounds presented to the auditory central nervous system dichotically [16]. Although the effects of sleep deprivation have been extensively studied in several paradigms, including sophisticated neuropsychological and imaging studies $[11,17,18]$, there are still many questions to be answered with respect to how brain circuits are integrated in healthy and adverse conditions. With the objective of contributing to the latter, we designed the present investigation to test the hypothesis that 24 hours of sleep deprivation would negatively affect CAP in otherwise healthy adults.

\section{Methods}

\section{Participants}

Inclusion criteria included the following: 18-40 years of age; physically and psychologically healthy, and good sleep habits. The exclusion criteria were: diagnosis of sleep disorders, hearing loss, or other neurological diseases; family history of hereditary diseases, as assessed by physical examination and history; and medication use within the 7 days preceding the initial hearing evaluation and baseline (BSL) CAP examination. Use of a medication during the period between the BSL and 24hSD test sessions would also result in exclusion from the study.

A total of 30 healthy volunteers met the inclusion criteria and agreed to participate in the study. The cohort included 17 females (56.7 \%) and 13 males (43.3\%), with a mean \pm standard deviation, age $30.7 \pm 7.1$ years. All participants indicated that they did not smoke, consume any medications, stimulants, caffeine, or alcohol for at least 24 hours prior to the BLS and 24hSD test sessions. We obtained informed formal written consent from all participating subjects, and the research project was approved by the Ethics Committee on Research Involving Human Subjects (registration number CEP 0527/08 at the Hospital Pequeno Principe, Curitiba, Brazil).

\section{Study design}

An initial hearing evaluation consisted of pure tone air $(250-8000 \mathrm{~Hz})$ and bone $(500-4000 \mathrm{~Hz})$ conduction threshold audiometry, a speech recognition threshold test, a speech recognition percent index, and acoustic impedance measurements. Preliminary audiometric evaluations included tympanometric curve measurements for which a type A tympanometric (tympanogram) finding (i.e., maximum impedance at or near $0 \mathrm{~Pa}$ ) indicated normal middle ear physiology and a type Ad tympanometric curve (i.e., an extremely high impedance peak) was taken as evidence of disjunction of the ossicular chain and/or abnormalities in the tympanic membrane.

CAP was assessed by the RGDT and the SSWT at two different times (BSL and 24hSD) with an inter-test interval of 2-3 months, during which the participants were allowed to continue to consume alcoholic or caffeinated beverages. The BSL CAP tests were conducted immediately after the initial hearing evaluation. The RGDT and the SSWT were conducted using a D88 discman, coupled to a Madsen Itera II diagnostic audiometer (GN Otometrics, Tasstrup, Denmark) with TDH39 headphones.

The first CAP session, BSL, was performed in a normal (no sleep deprivation) control condition and the second test was conducted in the 24hSD state following 24 hours of no sleep. In consideration of the participants' convenience and safety, SD was carried out on Fridays, and the 24hSD measurements were conducted on Saturdays. A research assistant remained with the subjects throughout the night to ensure that they did not fall asleep during the experimental sleep deprivation period. Most of subjects chose to watch TV during the sleep deprivation period. All testing was performed at the phonoaudiology clinic of the University of Tuiuti in Brazil.

\section{RGDT}

The RGDT, which is described in detail elsewhere $[19,20]$, produces time scores wherein a lesser time (lower score) indicates better performance. In our RGDT protocol, pairs of pure tones were presented in the following frequencies: $500 \mathrm{~Hz}, 1000 \mathrm{~Hz}, 2000 \mathrm{~Hz}$, and $4000 \mathrm{~Hz}$. The procedure was repeated for each frequency, resulting in a trial that included four single- 
frequency runs. The intervals between paired tones ranged from $0 \mathrm{~ms}$ to $40 \mathrm{~ms}$ (specifically, $0,2,5,10,15,20$, 25,30 , or $40 \mathrm{~ms}$ ), with the order of the intervals being random. The interval between the consecutive presentations of tone pairs was 4.5 seconds to allow an adequate response time for the participants.

The RGDT was scored for the smallest interval between paired tones for which the individual was able to identify the presence of two discrete stimuli. This identification assessment involved an assessment of gap detection, which reflects the ability to resolve time differences. Within three noise bursts (two of which were unbroken), the subject was asked to identify which one included a silent interval ("gap"). Each person responded to the stimuli verbally or by holding up one or two fingers to indicate whether s/he heard one or two tones. Normal gap detection threshold was considered to be 2-20 ms. A gap detection threshold $>20 \mathrm{~ms}$ was considered abnormal.

\section{SSWT}

The SSWT, also described in detail elsewhere [16], produces percentage scores wherein a greater percentage (higher score) indicates better performance. The participants were presented with spondaic words (words with two syllables of equal stress) in an overlapping fashion such the second syllable of the first spondaic word occurred at the same time as the first syllable of the second spondaic word. One ear (the leading ear) was presented with the first syllable of the first spondaic word in isolation (non-competing), followed by the second syllable of the first spondaic word in a dichotic mode (competing). The other ear (the lagging ear) was presented with the second spondaic word such that the first syllable was presented in dichotic mode (during presentation of the second syllable of the first spondaic word to the leading ear) and the second syllable presented in isolation. Each ear served as the leading ear for half of the test presentations. The stimuli were presented at an intensity of $50 \mathrm{~dB}$ (SL re:RT/PTA). The testing time was $8 \mathrm{~min}-$ utes. Error scores were calculated for each ear in both competing and non-competing modes.

\section{Statistical analysis}

Two-tailed paired student's $t$ test was used to compare data between the BSL and 24hSD conditions. Two-way analyses of variance (ANOVAs) were conducted to test whether there were any significant differences among the sexes. $\mathrm{P}<0.05$ was considered statistically significant. All data are reported as means \pm SDs (standard deviations).

\section{Results}

\section{Hearing ability of participants}

Preliminary audiometric evaluations using tympanometric curve measurements confirmed that all 30 participants had normal hearing. Most of the participants, 28/30 (93.4 \%), were found to have type A tympanometric curves bilaterally (maximum impedance at or near $0 \mathrm{~Pa}$ ); the remaining $2(6.7 \%)$ had type Ad tympanometric curves (with an extremely high impedance peak in the curve). Additionally, we found that 3/30 subjects $(10.0 \%)$ had a bilateral absence of the ipsilateral reflex, 1 (3.4\%) had an absence of the contralateral reflex on the right only, and $1(3.4 \%)$ had an absence in all frequencies tested. Most of the volunteers, 25/30 (83.4\%), had acoustic reflexes at all frequencies tested.

\section{SD worsens RGDT performance}

As shown in Figure 1, the mean RGDT score for the entire group in the non-sleep deprived BSL condition was $6.4 \pm 2.8 \mathrm{~ms}$ (range, $2.0-13.7 \mathrm{~ms}$ ), and was elevated to $8.0 \pm 2.9 \mathrm{~ms}$ (range, $2.7-13.7 \mathrm{~ms}$ ) in the $24 \mathrm{hSD}$ condition $(p=0.0005)$. Subsequent analysis of the RGDT data by gender subgroup showed that this effect was not gender dependent, as this pattern of results was replicated within both the male and the female subgroups. The mean RGDT score for males increased from $4.7 \pm 2.7 \mathrm{~ms}$ (range, 2.0-10.0 ms) in the BSL condition to $6.6 \mathrm{~ms} \pm 2.9 \mathrm{~ms}$ (range, $2.7-10.0 \mathrm{~ms})$ in the $24 \mathrm{hSD}$ condition $(p=0.0066)$. Likewise, the mean RGDT score for females increased from $7.7 \pm 2.4 \mathrm{~ms}$ (range, 5.0-13.7 $\mathrm{ms}$ ) in the BSL condition to $9.0 \pm 2.5 \mathrm{~ms}$ (range, $5.0-13.7 \mathrm{~ms}$ ) in the $24 \mathrm{hSD}$

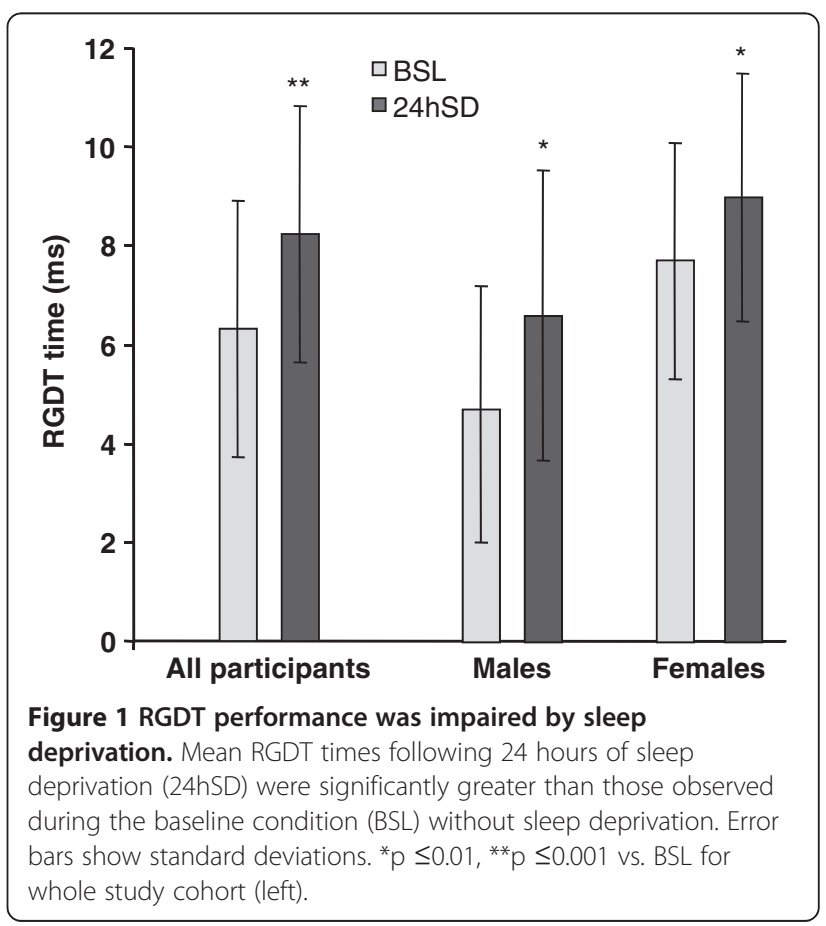


condition $(p=0.0208)$. Although both sex subgroups exhibited the aforementioned increases from BSL to the 24hSD condition, an ANOVA showed that males and females differed from each other at BSL $(p=0.0026)$ and in the 24hSD condition ( $p=0.0206)$.

\section{SD worsens SSWT performance}

As illustrated in Figure 2a and b, the study cohort as a whole showed significant worsening of performance in the SSWT in both the right ear and the left ear following sleep deprivation. In the right ear, the group mean fell from $98.4 \pm 1.8 \%$ (range, 92.0-100.0 \%) in the BSL

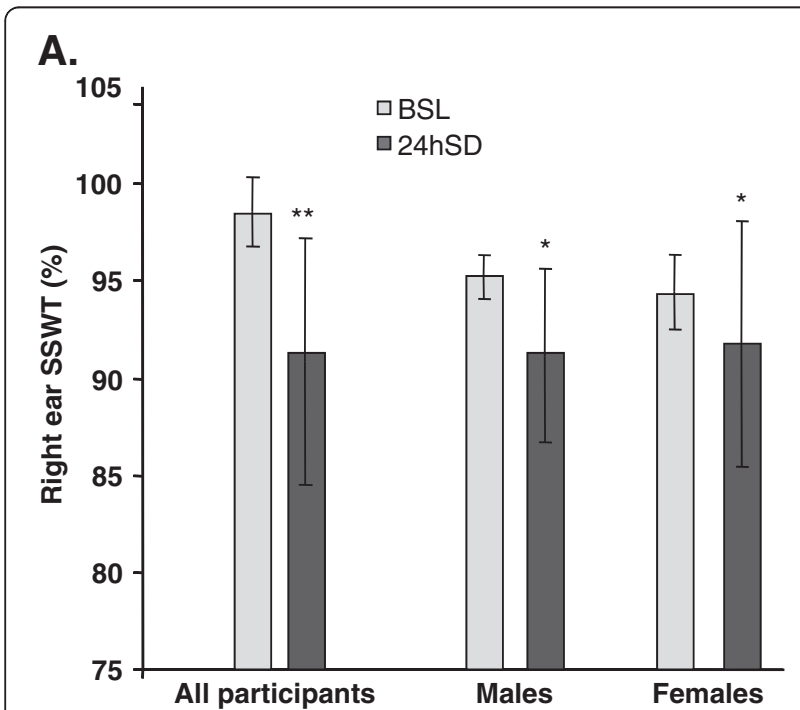

B.

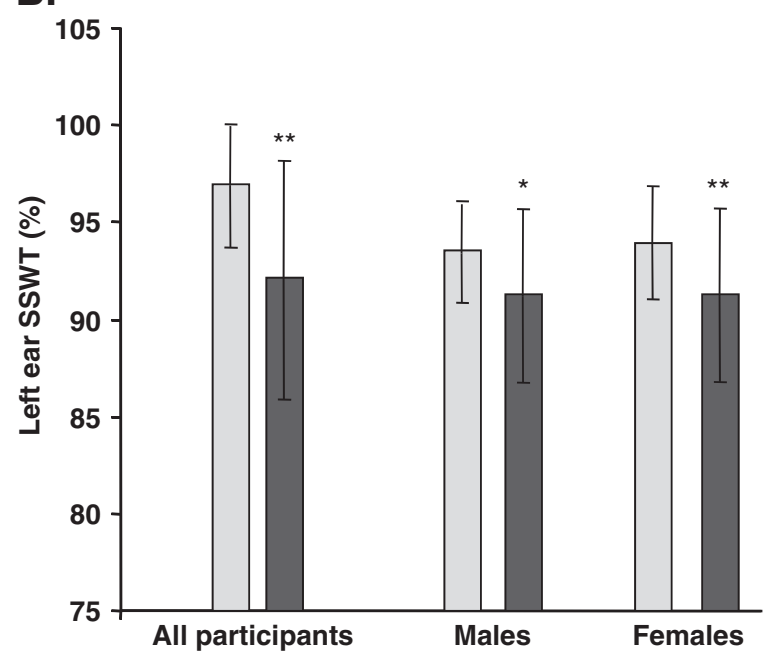

Figure 2 SSWT performance was impaired by sleep deprivation. Mean SSWT times following 24 hours of sleep deprivation (24hSD) were significantly reduced compared to those observed during the baseline condition (BSL) without sleep deprivation. Error bars show standard deviations. ${ }^{*} \mathrm{p} \leq 0.01$, ${ }^{* *} \mathrm{p} \leq 0.001$ vs. BSL for whole study cohort (left) or within gender subgroups. condition to $94.2 \pm 6.3 \%$ (range, 80.0-100.0 \%) in the $24 \mathrm{hSD}$ condition $(p=0.0005)$. In the left ear, the group mean fell from $96.7 \pm 3.1 \%$ (range, 90.0-100.0 \%) in the BSL condition to $92.1 \pm 6.1 \%$ (range, $80.0-100.0 \%$ ) in the 24hSD condition $(p<0.0001)$.

An ANOVA of the SSWT data by gender subgroup showed that the 24hSD effect on SSWT performance was not gender dependent (Fig. $2 \mathrm{a}$ and $\mathrm{b}$ ). In the right ear of males, the mean SSWT score fell from $98.5 \pm 1.3 \%$ (range, 97.5-100.0 \%) in the BSL condition to $93.9 \pm 5.2 \%$ (range, $85.0-100.0 \%$ ) in the $24 \mathrm{hSD}$ condition $(p=0.0080)$, and in the left ear of males, the mean SSWT score fell from $96.5 \pm 2.9 \%$ (range, 92.5-100.0 \%) in the BSL condition to $91.5 \pm 6.9 \%$ (range, 80.0$100.0 \%)$ in the $24 \mathrm{hSD}$ condition $(p=0.0076)$. Similarly, in the right ear of females, the mean SSWT score fell from $98.4 \pm 2.2 \%$ (range, 92.0-100.0 \%) in the BSL condition to $94.4 \pm 7.3 \%$ (range, 80.0-100.0 \%) in the 24hSD $(p=0.0143)$, while in the left ear of females, the mean SSWT score fell from 96.9 $\pm 3.4 \%$ (range, 90.0$100.0 \%$ ) in the BSL condition to $92.5 \pm 3.4 \%$ (range, 80.0-100.0 \%) in the 24hSD condition ( $p=0.0010)$. There were no gender differences in SSWT results at BSL or in the 24hSD condition ( $p>0.05)$.

\section{Discussion}

The overall aim of this study was to investigate the impact of sleep deprivation on CAP in a group of healthy adults by comparing performance in the RGDT and the SSWT in a BSL (rested) condition versus after 24 hours of sleep deprivation. We showed that this relatively short period of sleep deprivation significantly impaired CAP in both males and females. More specifically, the increase in the average time needed to identify the interval of silence between paired sound stimuli in the RGDT that was observed following sleep deprivation demonstrated a negative impact of sleep deprivation on temporal resolution ability. Additionally, the decrease in SSWT performance observed in the $24 \mathrm{hSD}$ condition relative to the BSL condition indicates that 24 hours of sleep deprivation impaired the participants' ability to transfer auditory information between the cerebral hemispheres via the posterior portions of the corpus callosum [21]. Although there were differences between males and females at BLS and at the 24hSD in the RGDT, the overall CAP impairments in male and female subjects followed a similar pattern, indicating that the effects of sleep deprivation on CAP are not related to gender.

Acute sleep deprivation has a major impact on brain neurophysiology and can trigger changes in cortical and subcortical structures. Notably, prolonged wakefulness can alter frontal lobe functioning, affecting language expression and perception, reducing one's ability to perform creative processes, pay attention, and concentrate 
[22]. The neurophysiological impairment of the lateral (neocortical) temporal lobe after acute sleep deprivation is manifested in changes of auditory abilities, including sound perception and the processing of auditory information [23]. On the other hand, sleep deprivationassociated neurophysiological changes in the medial temporal lobe structures, especially the hippocampal formation and amygdala, may impair memory (e.g., the ability to remember words) and may alter mood and behavior [22,24]. Sleep deprivation can also impair functioning of the posterior temporal and parietal regions, resulting in a decreased ability to identify the main focus of attention [25].

CAP functions are heavily dependent on alertness and concentration. Thus, acute sleep deprivation that temporarily affects attention could, consequently, also alter the phenomena that constitute the neurobiological bases of CAP. From a neuropsychological perspective- which predicts that all memory-based tasks should be affected by SD [13] - studies have shown that language tasks that require sustained attention and higher level processing, such as reading comprehension, were negatively affected by sleep deprivation, whereas other tasks that rely on basic language processing, such as order recall or category recall, were not affected [26]. Furthermore, some executive functions, the ability to execute complex tasks (i.e., creative problem solving), decision making, attention, and vigilance have been shown to be impaired by sleep loss $[27,28]$. However, other researchers have found that acute sleep deprivation did not impair performance in complex cognitive tasks that require critical thinking and reasoning [26].

The aforementioned findings [26] may seem paradoxical since the prefrontal cortex (PFC) is activated during attention, vigilance, and complex cognitive tasks. The fact that studies have shown that some cognitive tasks that could be considered stimulating and that require active and frequent responses were less affected by acute sleep deprivation [26] could be interpreted as indicating that sleep deprivation-resistant cognitive tasks, regardless of complexity or stimulation, could be activating the reward dopaminergic system, and thus overrunning the impairing effects of acute sleep deprivation. For instance, using functional magnetic resonance imaging, Libedinsky and colleagues [29] showed that when subjects under total sleep deprivation were presented with images that produced anticipation of monetary or social rewards, the ventromedial PFC was activated. The mesocorticolimbic brain reward circuit involves activation of the dopaminergic system, similar to a pharmacological stimulant. Such increases in dopamine (DA) release, especially in the PFC, could counteract some of the cognitive deficits associated with sleep deprivation [30].
The neurochemical mechanisms responsible for decreased performance in neurocognitive and attention tests after varying periods of sleep deprivation are not fully understood. However, some studies have suggested that DA may be responsible for the behavioral changes observed following sleep deprivation. Studies in rats have shown that brain levels of DA and noradrenaline are increased after 24 hours of sleep deprivation, peaking at 96 hours of sleep deprivation and recovering to normal levels after 24 hours of rest [31]. Imaging studies in humans have shown that DA release is significantly increased after 24 hours of total sleep deprivation [11]. Other studies have shown that, although the dopamine transporter (DAT) was not influenced by 4 nights of REM sleep deprivation and 2 nights of total sleep deprivation, the sleep deprived participants showed an increase in DAT density and it was positively correlated with estradiol concentration [32].

While, the implications of the above DA system findings have yet to be elucidated, we do know that DA plays a major role in attentional networks [33]. The ability to maintain attention for long periods of time has been linked to DA actions in the anterior frontal cortex [33]. The neural network associated with attention appears to involve the integrated operation of the prefrontal, temporal, and parietal cortices, the basal ganglia, and the cerebellum [34,35]. Topographically, the cortical regions associated with attentional focus partially overlap with the auditory association cortex. Thus, theoretically, it is possible that the attentional network could directly influence the ability to process auditory information. Indeed, recent research has shown that the neurophysiology of the frontal, temporal, and parietal lobes appears to be severely impaired after 24 hours of sleep deprivation; such impairments may consequently impair CAP [36].

Memories are essential to accurate processing of auditory information $[37,38]$. Although memory consolidation is a complex phenomena, a convergence of evidence suggests that sleep may play a critical role in memory consolidation [3]. Indeed, people commonly show impaired memory following acute sleep deprivation [3]. Vecsey and colleagues [39] documented decreased levels of adenosine 3,5-cyclic monophosphate (cAMP) in the hippocampal cells of sleep deprived rats; cAMP is a major second messenger involved in diverse biological phenomena, including memory consolidation [39].

We chose to use the RGDT in this study because auditory temporal resolution represents the neurophysiological basis of CAP. Temporal resolution allows us to understand human speech continuously. Thus, impairment of this ability may make it impossible for one to recognize discrete, meaningful variations in speech. Indeed, virtually all auditory information is influenced by the variable of time [40]. 
The literature is inconsistent with respect to how much sleep deprivation is needed to produce effects on verbal and executive cognitive processes. Some authors have suggested that sleep deprivation less than 40 hours in duration may not significantly affect complex cognitive processes [41,42], whereas other authors have reported findings of changes in mood, behavior, and attention after only 24 hours of continuous wakefulness [18]. Moreover, differential responses to sleep deprivation across different individuals may depend on several endogenous and exogenous factors, such as the period without sleep, circadian rhythms, and personal motivation, as well as behavioral and personality characteristics [1].

Our findings should be considered in light of some limitations. Firstly, we did not ask the participants to wear an activity meter (actimeter) or to complete a sleep $\log$ prior to testing. It should be noted however that sleep logs provide only subjective information of the participants' sleep habits. Moreover, since all of the participants were professionals with a college degree, and were willing to participate in study without any financial compensation, we assumed that they were honest and serious about our study.

Secondly, we did not explore in greater detail the gender differences observed in the RGDT condition. The main objective of the present study was not to investigate the presence of gender related differences. Nonetheless, some studies have documented gender related differences in cognitive performance and in visual and auditory processing of verbal and figural tasks; and sex differences in brain structure in auditory and cingulated regions have been reported [15,43-45]. Certainly, the gender factor could be further studied in a more controlled experiment with a larger number of participants.

Thirdly, since our sample was relatively small, we did not properly counterbalance for order. Ideally, half of the participants could have completed the BSL condition first and the 24hSD second, while the other half could have done the reverse. Finally, we did not collect any type of psychomotor vigilance data, reaction time data, or attentional measurements. Several studies have suggested that sleep deprivation has a major impact on sustained attention $[46,47]$. Thus, it could be that the observed sleep deprivation-related impairments in RGDT and SSWT performance were a result of brief attentional lapses rather than an impairment of CAP per se. Nevertheless, the results presented set the stage for further investigations and replications under more stringent experimental conditions.

\section{Conclusion}

In conclusion, the results presented here provide a demonstration that acute sleep deprivation for a period of 24 hours is sufficient to significantly worsen CAP in neurologically and psychologically healthy men and women. Impairment of CAP following sleep deprivation may be due the effects of extended wakefulness on neurobiological functions that are critical for the processing of auditory information, including memory, attention, concentration, reaction time, stimulus perception, and behavioral regulation.

\section{Abbreviations \\ ANOVA: Analysis of variance; BSL: Baseline; CAP: Central auditory processing: CAPD: Central auditory processing Disorder; DA: Dopamine; DAT: Dopamine transporter; REM: Rapid eye movement; RGDT: Random gap detection test; SD: Standard deviation; SSWT: Staggered spondaic word test.}

\section{Competing interests}

The authors declare that they have no competing interests.

\section{Author details}

'University Tuiuti of Paraná, Otoneurology Research Center, Curitiba, Brazil 2Department of Neuropediatrics, Little Prince Children's Hospital, Curitiba, Brazil. ${ }^{3}$ Department of Neuropsychopharmacology, Pelé Little Prince Research Institute, Curitiba, Brazil. ${ }^{4}$ Faculdades Little Prince, Curitiba, Brazil.

${ }^{5}$ Department of Psychiatry and Biobehavioral Sciences of the David Geffen School of Medicine, Semel Institute for Neuroscience and Human Behavior, University of California, Los Angeles, USA.

\section{Authors' contributions}

PBNL and ALJ were co-principal investigators of the study. PBNL, ALJ, and BSZ designed the study protocol. PBNL and KFKA collected the data. PBNL, BSZ, MLC, JMM and AL analyzed and interpreted the findings and wrote the manuscript. MLC performed a critical revision of the manuscript for important intellectual content and was responsible for submitting the final approved manuscript. JMM were responsible for the statistical analysis. All authors read and approved the final manuscript.

Received: 8 February 2012 Accepted: 23 July 2012

Published: 23 July 2012

\section{References}

1. Chokroverty S: Overview of sleep \& sleep disorders. Indian J Med Res 2010, 131:126-140.

2. Stickgold R, Hobson JA, Fosse R, Fosse M: Sleep, learning, and dreams: offline memory reprocessing. Science 2001, 294(5544):1052-1057.

3. Stickgold R, Walker MP: Sleep-dependent memory consolidation and reconsolidation. Sleep Med 2007, 8(4):331-343.

4. Bonnet MH, Arand DL: We are chronically sleep deprived. Sleep 1995, 18 (10):908-911.

5. Levine B, Lumley M, Roehrs T, Zorick F, Roth $T$ : The effects of acute sleep restriction and extension on sleep efficiency. Int J Neurosci 1988, 43(3-4):139-143.

6. Imeri L, Opp MR: How (and why) the immune system makes us sleep. Nat Rev Neurosci 2009, 10(3):199-210.

7. Robillard R, Lanfranchi PA, Prince F, Filipini D, Carrier J: Sleep deprivation increases blood pressure in healthy normotensive elderly and attenuates the blood pressure response to orthostatic challenge. Sleep 2011, 34(3):335-339.

8. Silva JA, Costa E: Sleep disorders in psychiatry. Metabolism 2006, 55(10 Suppl 2):S40-S44.

9. Kyung Lee E, Douglass AB: Sleep in psychiatric disorders: where are we now? Can J Psychiatry 2010, 55(7):403-412.

10. Mirescu C, Peters JD, Noiman L, Gould E: Sleep deprivation inhibits adult neurogenesis in the hippocampus by elevating glucocorticoids. Proc Natl Acad Sci USA 2006, 103(50):19170-19175.

11. Volkow ND, Wang GJ, Telang F, Fowler JS, Logan J, Wong C, Ma J, Pradhan K, Tomasi D, Thanos PK, et al: Sleep deprivation decreases binding of [11 C]raclopride to dopamine D2/D3 receptors in the human brain. J Neurosci 2008, 28(34):8454-8461.

12. Friederici AD: Towards a neural basis of auditory sentence processing. Trends Cogn Sci 2002, 6(2):78-84. 
13. Harrison Y, Horne JA: The impact of sleep deprivation on decision making: a review. J Exp Psychol Appl 2000, 6(3):236-249.

14. Harrison $Y$, Horne JA: Sleep loss and temporal memory. Q J Exp Psychol A 2000, 53(1):271-279.

15. Babkoff $H$, Zukerman G, Fostick L, Ben-Artzi E: Effect of the diurnal rhythm and $24 \mathrm{~h}$ of sleep deprivation on dichotic temporal order judgment. J Sleep Res 2005, 14(1):7-15.

16. Katz J, Smith PS: The Staggered Spondaic Word Test A ten-minute look at the central nervous system through the ears. Ann N Y Acad Sci 1991, 620:233-251.

17. Harrison Y, Horne JA, Rothwell A: Prefrontal neuropsychological effects of sleep deprivation in young adults-a model for healthy aging? Sleep 2000, 23(8):1067-1073.

18. Tomasi D, Wang RL, Telang F, Boronikolas V, Jayne MC, Wang GJ, Fowler JS, Volkow ND: Impairment of attentional networks after 1 night of sleep deprivation. Cereb Cortex 2009, 19(1):233-240.

19. Randon Gap Detection Test. [www.auditec.com]

20. Keith RW, Katbamna B, Tawfik S, Smolak LH: The effect of linguistic background on staggered spondaic word and dichotic consonant vowel scores. Br J Audiol 1987, 21(1):21-26.

21. Palfery TD, Duff D: Central auditory processing disorders: review and case study. Axone 2007, 28(3):20-23.

22. Paavonen EJ, Raikkonen $\mathrm{K}$, Pesonen AK, Lahti J, Komsi N, Heinonen K, Jarvenpaa AL, Strandberg T, Kajantie E, Porkka-Heiskanen T: Sleep quality and cognitive performance in 8-year-old children. Sleep Med 2010, 11(4):386-392.

23. Ayalon L, Ancoli-Israel S, Klemfuss Z, Shalauta MD, Drummond SP: Increased brain activation during verbal learning in obstructive sleep apnea. Neurolmage 2006, 31(4):1817-1825.

24. Beebe DW, Rose D, Amin R: Attention, learning, and arousal of experimentally sleep-restricted adolescents in a simulated classroom. $J$ Adolesc Health 2010, 47(5):523-525.

25. Chee MW, Choo WC: Functional imaging of working memory after $24 \mathrm{hr}$ of total sleep deprivation. J Neurosci 2004, 24(19):4560-4567.

26. Pilcher JJ, Band D, Odle-Dusseau HN, Muth ER: Human performance under sustained operations and acute sleep deprivation conditions: toward a model of controlled attention. Aviat Space Environ Med 2007, 78(5 Suppl): B15-B24.

27. Adam M, Retey JV, Khatami R, Landolt HP: Age-related changes in the time course of vigilant attention during 40 hours without sleep in men. Sleep 2006, 29(1):55-57.

28. Tucker AM, Whitney P, Belenky G, Hinson JM, Van Dongen HP: Effects of sleep deprivation on dissociated components of executive functioning. Sleep 2010, 33(1):47-57.

29. Libedinsky C, Smith DV, Teng CS, Namburi P, Chen WW, Huettel SA, Chee $\mathrm{MW}$ : Sleep deprivation alters valuation signals in the ventromedial prefrontal cortex. Front Behav Neurosci 2011, 5:70.

30. Boutrel B, Koob GF: What keeps us awake: the neuropharmacology of stimulants and wakefulness-promoting medications. Sleep 2004, 27 (6):1181-1194

31. Andersen ML, Martins PJ, D'Almeida V, Bignotto M, Tufik S: Endocrinological and catecholaminergic alterations during sleep deprivation and recovery in male rats. J Sleep Res 2005, 14(1):83-90.

32. Martins RC, Andersen ML, Garbuio SA, Bittencourt LR, Guindalini C, Shih MC, Hoexter MQ, Bressan RA, Castiglioni ML, Tufik S: Dopamine transporter regulation during four nights of REM sleep deprivation followed by recovery-an in vivo molecular imaging study in humans. Sleep 2010, 33(2):243-251.

33. Barkley RA, Grodzinsky G, DuPaul GJ: Frontal lobe functions in attention deficit disorder with and without hyperactivity: a review and research report. J Abnorm Child Psychol 1992, 20(2):163-188.

34. Brennan AR, Arnsten AF: Neuronal mechanisms underlying attention deficit hyperactivity disorder: the influence of arousal on prefrontal cortical function. Ann N Y Acad Sci 2008, 1129:236-245.

35. Petersen SE, Posner Ml: The attention system of the human brain: 20 years after. Annu Rev Neurosci 2012, 35:73-89.

36. Wu JC, Gillin JC, Buchsbaum MS, Chen P, Keator DB, Khosla Wu N, Darnall LA, Fallon JH, Bunney WE: Frontal lobe metabolic decreases with sleep deprivation not totally reversed by recovery sleep. Neuropsychopharmacology 2006, 31(12):2783-2792.
37. Quintas VG, Attoni TM, Keske-Soares M, Mezzomo CL: Auditory processing and phonological awareness in children with normal and deviant speech development. Pro Fono 2010, 22(4):497-502.

38. Steenari MR, Vuontela V, Paavonen EJ, Carlson S, Fjallberg M, Aronen E: Working memory and sleep in 6- to 13-year-old schoolchildren. J Am Acad Child Adolesc Psychiatry 2003, 42(1):85-92.

39. Vecsey CG, Baillie GS, Jaganath D, Havekes R, Daniels A, Wimmer M, Huang $T$, Brown KM, Li XY, Descalzi G, et al: Sleep deprivation impairs CAMP signalling in the hippocampus. Nature 2009, 461(7267):1122-1125.

40. Samelli $A G$, Schochat $E$ : The Gap-in-noise test: gap detection thresholds in normal hearin young adults. Int J Audiol 2008, 47(5):238-245.

41. Killgore WD: Effects of sleep deprivation on cognition. Prog Brain Res 2010, 185:105-129.

42. Kopasz M, Loessl B, Valerius G, Koenig E, Matthaeas N, Hornyak M, Kloepfer C, Nissen C, Riemann D, Voderholzer U: No persisting effect of partial sleep curtailment on cognitive performance and declarative memory recall in adolescents. J Sleep Res 2010, 19(1 Pt 1):71-79.

43. Brun CC, Lepore N, Luders E, Chou YY, Madsen SK, Toga AW, Thompson PM: Sex differences in brain structure in auditory and cingulate regions. Neuroreport 2009, 20(10):930-935.

44. Shively CA, Bethea CL: Cognition, mood disorders, and sex hormones. ILAR J 2004, 45(2):189-199.

45. Wallentin M: Putative sex differences in verbal abilities and language cortex: a critical review. Brain Lang 2009, 108(3):175-183.

46. Dinges DF, Pack F, Williams K, Gillen KA, Powell JW, Ott GE, Aptowicz C, Pack Al: Cumulative sleepiness, mood disturbance, and psychomotor vigilance performance decrements during a week of sleep restricted to 4-5 hours per night. Sleep 1997, 20(4):267-277.

47. Lim J, Dinges DF: Sleep deprivation and vigilant attention. Ann N Y Acad Sci 2008, 1129:305-322.

doi:10.1186/1471-2202-13-83

Cite this article as: Liberalesso et al: Effects of sleep deprivation on central auditory processing. BMC Neuroscience 2012 13:83

\section{Submit your next manuscript to BioMed Central and take full advantage of:}

- Convenient online submission

- Thorough peer review

- No space constraints or color figure charges

- Immediate publication on acceptance

- Inclusion in PubMed, CAS, Scopus and Google Scholar

- Research which is freely available for redistribution 